\title{
Does sleep-disordered breathing add to impairments in academic performance and brain structure usually observed in children with overweight/obesity?
}

\author{
Lucia V. Torres-Lopez ${ }^{1}$ (1) . Cristina Cadenas-Sanchez ${ }^{1,2}$. Jairo H. Migueles ${ }^{3,1,4}$. Irene Esteban-Cornejo' . \\ Pablo Molina-Garcia ${ }^{1}$. Charles H. Hillman ${ }^{5}$. Andres Catena ${ }^{6}$. Francisco B. Ortega $a^{1,7,4}$
}

Received: 4 October 2021 / Revised: 26 January 2022 / Accepted: 29 January 2022 / Published online: 10 February 2022

(c) The Author(s) 2022

\begin{abstract}
Approximately 4-11\% of children suffer from sleep-disordered breathing (SDB), and children with obesity are at increased risk. Both obesity and SDB have been separately associated with poorer brain health, yet whether SDB severity affects brain health in children with obesity remains unanswered. This study aimed to examine associations of SDB severity with academic performance and brain structure (i.e., total brain and gray and white matter volumes and gray matter volume in the hippocampus) in children with overweight/obesity. One hundred nine children aged 8-12 years with overweight/obesity were included. SDB severity and its subscales (i.e., snoring, daytime sleepiness, and inattention/hyperactivity) were evaluated via the Pediatric Sleep Questionnaire (PSQ), and academic performance was evaluated with the Woodcock-Muñoz standardized test and school grades. Brain structure was assessed by magnetic resonance imaging. SDB severity was not associated with academic performance measured by the standardized test (all $|\beta|>0.160, P>0.076$ ), yet it was associated with the school grade point average $(\beta=-0.226, P=0.007)$ and natural and social science grades $(\beta=-0.269, P=0.024)$. Intention/hyperactivity seemed to drive these associations. No associations were found between SDB severity and the remaining school grades (all $\beta<-0.188, P>0.065$ ) or brain volumes (all $P>0.05$ ).

Conclusion: Our study shows that SDB severity was associated with lower school grades, yet it was not associated with the standardized measurement of academic performance or with brain volumes in children with overweight/obesity. SDB severity may add to academic problems in children beyond the effects contributed by overweight/obesity status alone.

\section{What is Known:}

- Sleep-disordered breathing (SDB) may affect brain structure and academic performance in children.

- Children with overweight/obesity are at higher risk for the development of SDB, yet the comorbid obesity-SDB relationship with brain health has not been investigated thus far.

What is New:

- To our knowledge, this is the first study examining the associations of comorbid obesity-SDB severity with brain volumes and academic performance in children.

- SDB symptoms may adversely affect academic performance at school in children with overweight/obesity, beyond the effects of weight status alone.
\end{abstract}

Keywords Preadolescents $\cdot$ Childhood obesity $\cdot$ Obstructive sleep apnea $\cdot$ Academic achievement $\cdot$ Brain health

\section{Communicated by Gregorio Paolo Milani}

Lucia V. Torres-Lopez

luciatl@ugr.es

Francisco B. Ortega ortegaf@ugr.es

Extended author information available on the last page of the article

\section{Abbreviations}

GMV Gray matter volume

OSA Obstructive sleep apnea

SDB Sleep-disordered breathing

SRBD Sleep-related breathing disorders

WMV White matter volume 


\section{Introduction}

Pediatric obstructive sleep-disordered breathing (SDB) has been recognized as a common health problem [1]. The European Respiratory Society Task Force defined obstructive SDB as a set of respiratory tract dysfunctions during sleep, which are characterized by snoring and/or increased effort to breathe caused by a narrowing of the pharynx and increased airway resistance [2]. SDB affects approximately $4-11 \%$ of children, with a range of severities from primary snoring to obstructive sleep apnea (OSA) [3, 4]. In a previous study including children $6-15$ years of age, those with obesity were at higher risk for SDB (20.8\%) than their normal-weight peers (6.3\%) [4]. Among children with SDB, obesity has been associated with higher SDB severity $[5,6]$. These data clearly support the idea that there is an urgent need to address SDB severity in children with overweight/obesity.

A consensus statement by the European Respiratory Society Task Force on the diagnosis and management of SDB in childhood concluded that children with SDB may present an increased prevalence of academic difficulties and cognitive deficits [2]. Relatedly, some previous studies have found that children with SDB might present impaired academic [7-10] and neurocognitive $[11,12]$ performance and that SDB in children as young as 3 years old predicted significantly poorer performance on oral language assessments at 8 years old [13]. However, these findings have been inconsistent [11]. Specifically, research in children and adolescents with overweight/obesity and comorbid SDB has shown lower neurocognitive performance $[14,15]$ and contradictory findings on academic performance [16-19]. Thus, children with overweight/obesity may be at increased risk of neurocognitive deficits when they present with SDB symptoms $[14,15$, 20]. However, more research is needed to better understand the associations with academic performance.

Childhood is an important period for the development and maturation of the brain [21,22], and the hippocampus is a key subcortical structure involved in memory consolidation during wakefulness and sleep [23, 24]. Among the studies investigating pediatric SDB-brain structure links [25-32], only a few found significant associations in the hippocampus [29, 31, 33]. Beyond the hippocampus, a recent review concluded that obstructive SDB in children was associated with magnetic resonance imaging (MRI)assessed brain structure [34]. For example, children with OSA presented smaller regional gray matter volume (GMV) in areas related to cognitive control (i.e., frontal and prefrontal cortices, parietal cortices, temporal lobes, and brainstem) than their peers without any sleep disorder [32]. Conversely, other studies have shown no clear evidence of an association between OSA and brain structure [35, 36]. Regarding children with obesity, they have been shown to more prone to suffer from OSA than children with normal weight [37], and they often present impaired regional gray and white matter development [38]. It remains to be elucidated whether SDB increases brain structure impairments in children with overweight/obesity.

Evidence is lacking regarding the joint role of SDB and obesity on academic performance and brain structure in children. Identifying the associations of SDB severity with academic performance and brain structure in the context of childhood obesity could provide meaningful information for public health systems to prevent or attenuate the healthrelated consequences of obesity with efficient interventions. Therefore, the present study investigated the associations of SDB severity with academic performance using two different measures (i.e., standardized tests and school grades) and with brain structure (i.e., total brain volumes and hippocampal volume as a primary region of interest) in children with overweight/obesity. Identifying these associations might help to provide a better understanding of the influencing role of SDB on brain health beyond weight status alone.

\section{Methods}

\section{Study design and participants}

We used baseline data from ActiveBrains, a randomized controlled trial intended to investigate the effects of an exercise program on the brain and cognition in children with overweight/obesity. Data were collected from November 2014 to February 2016 in a total of 110 overweight/obese children from Granada (Spain). Of them, one child was excluded for being a nonnative Spanish speaker and did not reach the required threshold in Spanish reading comprehension to be properly evaluated. Thus, a total of 109 children $(10.04 \pm 1.12$ years old; 45 girls) were included in this crosssectional study. Most of the participants were European Caucasian (95\%), with only 5\% reported being of other ethnicities. A detailed description of the study protocol, aims, and methods has been published elsewhere [39]. All participants met the following inclusion criteria: (1) overweight or obesity, based on the sex- and age-specific international body mass index cutoff points proposed by the World Obesity Federation [40, 41]; (2) age between 8 and 12 years old; (3) no physical disabilities or neurological disorders that could affect physical performance; (4) prepubertal status according to the Tanner stages [42]; and (5) for the girls, no menarche.

Legal guardians were informed of the purpose of the study, and written informed consent for all participants was obtained. This study was conducted in accordance with the Declaration of Helsinki. The study protocol was approved 
by the Ethics Committee on Human Research (CEIH) of the University of Granada and registered at ClinicalTrials.gov (identifier: NCT02295072).

\section{Procedures and measurements}

\section{Sleep-disordered breathing}

The children's legal guardians completed the Spanish version of the Pediatric Sleep Questionnaire (PSQ) to assess SDB, which has shown high reliability and internal consistency [43]. This questionnaire has been validated for the identification of SDB [44, 45]. A sleep-related breathing disorders (SRBD) scale (scores could range from 0 to 1 ) was calculated from the 22 items of the questionnaire, with higher scores indicating greater severity [45]. The SRBD scale consisted of 22 closed response question items as a reduced version of the PSQ, which were divided into the following three subscales $[44,45]$ : snoring (four items), sleepiness (four items), and inattention/hyperactivity (six items). These subscales are valid and reliable for assessing SDB in children [44]. The answer options for each item included "yes," "no," and "I do not know." In the last domain of the questionnaire (inattention/hyperactivity subscale), the answers were structured as "never," "sometimes," "often," or "almost always." To be consistent with these answer options throughout the questionnaire, one of the sections was recategorized as follows: "never" and "sometimes" answers were categorized as "no," whereas "often" and "almost always" answers were categorized as "yes." The overall scale was calculated as the sum of the affirmative answers divided by 22 . Occasional missing answers or responses of "I do not know" were discounted from the denominator when calculating these proportions [46]. Scores $>0.33$ were considered suggestive of high risk for pediatric SDB $[44,45]$. A recent meta-analysis indicated that the SRBD scale was accurate for detecting OSA in children [47]. The SRBD scale score has been validated as a continuous indicator of SDB severity [6].

\section{Academic performance}

Academic performance was assessed via the Spanish adaptation of the Woodcock-Johnson III battery (i.e., WoodcockMuñoz standardized test), which has shown high reliability and is a well-validated measure of academic performance for individuals aged 5-95 years [48]. For this study, we used the standard battery, which contained three tests of mathematics, three tests of reading, and three tests of written language. The assessment was individually conducted for each child by a trained evaluator in a session lasting 100-120 min. The data registered for each child were individually checked by two trained evaluators. All data were processed using Compuscore and Profile software version 3.1. (Riverside Publishing Company, Itasca, IL, USA) and grouped into components (i.e., total achievement, reading, writing, written expression, mathematics, calculation, and calculation skills). For the current study, a standard T-score based on an average of 100 and a standard deviation of 15 was obtained for the following academic performance indicators: mathematics, reading, writing, and total achievement. Academic performance tests and MRI were conducted within 1 to 3 weeks of each other.

As a second measure of academic performance, official final school grades, closer to the baseline assessment ( \pm 1.5 months), were used for the analyses in $78 \%$ of the study sample $(n=85)$. Data were not missing at random $(n=24)$. The school grades were initially collected in the second wave of participants, so they were missing for the first wave. Since school records were provided in a qualitative manner (i.e., insufficient, sufficient, good, very good, and outstanding) by teachers, we registered the grades based on a scale from 1 to 5. The mean grades in mathematics, Spanish language, English language, and natural and social sciences were recorded. We also determined the individual's grade point average as the mean score across all subjects.

\section{Brain structure}

Brain volume was assessed using magnetic resonance imaging (Siemens Magnetom Tim Trio, 3 T, Siemens, Erlangen, Germany) equipped with a 32-channel head coil. High-resolution, T1-weighted images were acquired using a 3D MPRAGE (magnetization-prepared rapid gradientecho) protocol. Acquisition parameters were as follows: repetition time $=2,300 \mathrm{~ms}$, echo time $=3.1 \mathrm{~ms}$, inversion time $=900 \mathrm{~ms}$, flip angle $=9^{\circ}$, field of view $=256 \times 256$, acquisition matrix $=320 \times 320,208$ slices, and resolution $=0.8 \times 0.8 \times 0.8 \mathrm{~mm}$. The total acquisition time for the $\mathrm{T} 1$ sequence was $7 \mathrm{~min}$ and $31 \mathrm{~s}$ for each child. Imaging preprocessing included quality control, motion correction, spatial normalization to an MNI (Montreal Neurological Institute) template, and spatial smoothing. Detailed information about preprocessing has been described previously [49]. Some missing data were found for brain volumes $(n=9)$ and hippocampal GMV $(n=7)$. Total brain volume, total white matter volume (WMV), total GMV, and total right and left hippocampal GMV were derived from FreeSurfer software version 5.3.0 (Laboratory for Computational Neuroimaging, Athinoula A. Martinos Center for Biomedical Imaging, Harvard Medical School, Boston MA, USA). 


\section{Covariates}

Age, sex, maternal and paternal education levels, wave of participation (i.e., ActiveBrains was conducted in three waves for logistic reasons, including seasonality and differences between participants from different waves that may affect the associations), and peak height velocity (PHV) were considered potential confounders. Maternal and paternal maximum education levels were obtained by a self-report questionnaire. The responses of the mothers and fathers were classified as primary school, secondary school, and university degree completed. The wave of participation $(1,2$, or 3 ) was categorized into two dichotomous dummy variables. PHV is an indicator of maturity during childhood and adolescence calculated from standing and seated height using Moore's equations [50].

\section{Statistical analysis}

Descriptive characteristics are presented as the means and standard deviations for continuous variables and as frequencies and percentages for categorical variables. Sex differences were examined by $t$ tests and chi-square tests. All variables were checked for normality of distribution using the Kolmogorov-Smirnov test and visual inspection of histograms. After checking for a normal distribution, the SRBD scale and subscales were normalized using Blom's formula [51] because the distributions were skewed.

Hierarchical linear regression analyses were performed to examine the associations of SDB severity with academic performance and brain structure indicators (i.e., total GMV and WMV, total brain volume, and right and left hippocampal GMV). The stepwise method was used to investigate the influence of potential confounders in the models in step 1 (i.e., age, sex, maternal education level, paternal education level, wave of participation, and PHV). Next, hierarchical regressions were carried out, entering the SRBD scale scores as a predictor in step 2 and each academic performance and brain structure variable as outcomes in separate regression analyses after the inclusion of confounders previously defined in step 1 . The same procedure was followed to assess the association of each subscale score from the PSQ (i.e., snoring, daytime sleepiness, inattention/hyperactivity) with academic achievement and brain structure.

The Benjamini-Hochberg procedure was applied to account for random effects in multiple comparisons for each dependent domain (i.e., academic performance assessed by standardized test, academic performance assessed by school grades, brain volumes, and hippocampal GMV) with $q=0.1$ [52]. All analyses were performed using the Statistical Package for Social Science (IBM
SPSS Statistics for Windows, version 22.0, Armonk, NY). The level of significance was set at $p<0.05$.

\section{Results}

Descriptive characteristics for all participants are presented in Table 1. The results were not reported separately by sex since no significant sex interaction was observed in the models (sex was included as a confounder). Hierarchical linear regression analyses for the association of SDB severity with academic performance and brain structure variables are presented in Table 2. Regarding academic performance measured by standardized testing, SDB severity was not significantly associated with mathematics, reading, writing, or total academic performance (all $|\beta|>0.160, P \geq 0.076$ ). Regarding school grades, SDB severity was significantly associated with natural and social sciences grades and grade point average $(\beta=-0.226$, $P=0.024 ; \beta=-0.269, P=0.007$, respectively). Nonsignificant associations were found between SDB severity and mathematics, Spanish language and English language (all $\beta<-0.188, P>0.065$ ), and SDB severity was not associated with any of the brain outcome measures studied (all $P>0.05)$. Sensitivity analyses were performed by adding BMI as a confounder to previous models, and all associations presented in this study remained unchanged (data not shown). Additionally, hierarchical linear regression analyses were performed to assess potential associations of SDB subscale scores (snoring, sleepiness and inattention/hyperactivity) with academic performance and brain structure, as shown in Table 3. The PSQ inattention/hyperactivity subscale was the only subscale associated with school grades (specifically with mathematics grades, natural and social science grades, and grade point average; all $P<0.014)$. No significant differences were observed in the remaining aspects of academic performance and brain structure variables examined.

\section{Discussion}

The main findings of this study support the ideas that (1) SDB severity was related to lower academic performance at school, particularly with natural and social science grades and grade point average, but not with academic performance measured by a standardized test; (2) inattention/hyperactivity seemed to drive these associations between SDB severity and school grades; and (3) SDB severity was not associated with differences in brain structure in children with overweight/ obesity. Therefore, this study adds to the literature by showing that SDB symptoms may worsen academic performance in children beyond the effects of weight status alone. 
Table 1 Descriptive characteristics of the participants

\begin{tabular}{|c|c|c|c|c|c|c|c|}
\hline & & All & & Boys & & Girls & $P$ \\
\hline & $\mathrm{N}$ & Mean \pm SD & $\mathrm{N}$ & Mean \pm SD & $\mathrm{N}$ & Mean \pm SD & \\
\hline Age (years) & 109 & $10.04 \pm 1.12$ & 64 & $10.16 \pm 1.15$ & 45 & $9.88 \pm 1.08$ & 0.197 \\
\hline Weight (kg) & 109 & $56.21 \pm 11.23$ & 64 & $57.11 \pm 11.2$ & 45 & $54.94 \pm 11.28$ & 0.323 \\
\hline Height (cm) & 109 & $144.22 \pm 8.41$ & 64 & $144.98 \pm 7.97$ & 45 & $143.13 \pm 8.99$ & 0.261 \\
\hline BMI categories ${ }^{a}$ & 109 & & & & & & 0.606 \\
\hline Overweight, $n(\%)$ & & $28(25.7)$ & & $16(25)$ & & $12(26.7)$ & \\
\hline Obesity class I, $n(\%)$ & & $47(43.1)$ & & $30(46.9)$ & & $17(37.8)$ & \\
\hline Obesity class II, $n(\%)$ & & $34(31.2)$ & & $18(28.1)$ & & $16(35.6)$ & \\
\hline BMI z-scores ${ }^{b}$ & 107 & $3.04 \pm 0.89$ & 63 & $3.22 \pm 1.00$ & 44 & $2.80 \pm 0.63$ & 0.008 \\
\hline \multicolumn{8}{|c|}{ Sleep-related breathing disorders (SRBD) } \\
\hline SRBD scale (range: 0 to 1 ) & 109 & $0.19 \pm 0.13$ & 64 & $0.19 \pm 0.13$ & 45 & $0.18 \pm 0.13$ & 0.621 \\
\hline SRBD presence, $n(\%)$ & & $17(15.6)$ & & $10(15.6)$ & & $7(15.6)$ & 0.992 \\
\hline \multicolumn{8}{|c|}{ Academic performance $\left(\right.$ Woodcock-Muñoz) ${ }^{c}$} \\
\hline Mathematics & 109 & $102.06 \pm 10.91$ & 65 & $102.66 \pm 11.72$ & 44 & $101.18 \pm 9.64$ & 0.490 \\
\hline Reading & 109 & $108.46 \pm 12.68$ & 65 & $108.83 \pm 11.02$ & 44 & $107.91 \pm 14.91$ & 0.711 \\
\hline Writing & 109 & $114.1 \pm 12.84$ & 65 & $112.98 \pm 12.13$ & 44 & $115.75 \pm 13.8$ & 0.272 \\
\hline Total achievement & 109 & $109.56 \pm 11.83$ & 65 & $109.51 \pm 11.07$ & 44 & $109.64 \pm 13.01$ & 0.956 \\
\hline \multicolumn{8}{|c|}{ Academic performance (school grades) range: 0 to 5} \\
\hline Mathematics & 85 & $3.69 \pm 1.01$ & 51 & $3.73 \pm 1.01$ & 34 & $3.63 \pm 1.01$ & 0.642 \\
\hline Spanish language & 85 & $3.72 \pm 0.97$ & 51 & $3.61 \pm 0.93$ & 34 & $3.88 \pm 1.03$ & 0.211 \\
\hline English language & 85 & $3.65 \pm 1.12$ & 51 & $3.51 \pm 1.14$ & 34 & $3.86 \pm 1.08$ & 0.157 \\
\hline Natural and social science & 85 & $3.71 \pm 1.02$ & 51 & $3.64 \pm 1.11$ & 34 & $3.81 \pm 0.87$ & 0.424 \\
\hline Grade point average & 85 & $3.77 \pm 0.79$ & 51 & $3.69 \pm 0.82$ & 34 & $3.9 \pm 0.73$ & 0.218 \\
\hline \multicolumn{8}{|l|}{ Brain volumes $\left(\mathrm{mm}^{3}\right)$} \\
\hline Total gray matter & 100 & $793.48 \pm 66.22$ & 60 & $819.49 \pm 56.13$ & 40 & $754.48 \pm 61.36$ & $<0.001$ \\
\hline Total white matter & 100 & $406.81 \pm 47.97$ & 60 & $426.88 \pm 42.86$ & 40 & $376.7 \pm 38.9$ & $<0.001$ \\
\hline Total brain volume & 100 & $1200.29 \pm 106.69$ & 60 & $1246.37 \pm 88.88$ & 40 & $1131.18 \pm 93.71$ & $<0.001$ \\
\hline \multicolumn{8}{|l|}{ Hippocampal GMV } \\
\hline Right hippocampus & 102 & $3582.42 \pm 390.34$ & 60 & $3675.44 \pm 386.45$ & 42 & $3449.54 \pm 360.11$ & 0.004 \\
\hline Left hippocampus & 102 & $3454.5 \pm 379.77$ & 60 & $3522.31 \pm 387.57$ & 42 & $3357.62 \pm 350.42$ & $\mathbf{0 . 0 3 0}$ \\
\hline Total hippocampus & 102 & $7036.92 \pm 694.08$ & 60 & $7197.75 \pm 666.26$ & 42 & $6807.16 \pm 675.3$ & 0.005 \\
\hline
\end{tabular}

Data shown are mean \pm standard deviation or $n, \%$. Differences between sexes were examined by $t$-test or chi-square test. Statistically significant values $(P<0.05)$ are shown in bold. Notes: $S D$ standard deviation. ${ }^{a}$ BMI categories (overweight, obesity class I and obesity class II) were based on the sex- and age-specific international BMI cut-points proposed by the World Obesity Federation (https://www.euro.who.int/en/health-topics/ disease-prevention/nutrition/a-healthy-lifestyle/body-mass-index-bmi). ${ }^{b}$ BMI z-score based on World Health Organization. ${ }^{\mathrm{c}}$ Academic performance indicators were calculated based on standardized scores centered at 100 . Standardized composite scores are presented. $B M I$ body mass index, $S D B$ sleep-disordered breathing, $S R B D$ sleep-related breathing disorders, $G M V$ gray matter volume

Our study findings suggest a lower academic performance in children with a high risk of SDB than in their peers with a low risk of SDB. It may be that a single assessment through the administration of a standardized test might not fully capture the performance of children across time in the same manner as school grades do. Other possible factors explaining the differences between the school grade and standardized test outcomes may be the different grading systems between schools or the subjective influence of teachers [53]. To the best of our knowledge, only a few studies have focused on children with overweight/obesity with comorbid SDB and academic performance. Collectively, the conclusions across studies have been inconsistent [16-19]. In line with our findings, a previous study showed that academic grades were impaired in children with overweight and SDB compared to children with overweight but without SDB based on reports from parents and children [18]. Beebe et al. [18] found that these associations might be explained by behavioral factors rather than cognitive factors, which may explain why we observed associations with school grades but not with a standardized test. However, other studies measuring other dimensions of school functioning did not observe an impairment in children with SDB compared to children without SDB [19]. This inconsistency might be 
Table 2 Hierarchical linear regression for the associations of SDB severity with academic performance, brain volumes, and hippocampal gray matter volume variables

\begin{tabular}{|c|c|c|c|c|}
\hline & \multicolumn{4}{|c|}{ SRBD scale } \\
\hline & $\boldsymbol{\beta}$ & $R^{2}$ & CI & $P$ \\
\hline \multicolumn{5}{|c|}{ Academic performance (Woodcock-Muñoz) } \\
\hline Mathematics $\dagger$ & 0.023 & 0.228 & $(-0.150,0.196)$ & 0.793 \\
\hline Reading $\neq$ & -0.030 & 0.253 & $(-0.198,0.139)$ & 0.729 \\
\hline Writing & -0.160 & 0.177 & $(-0.338,0.017)$ & 0.076 \\
\hline Total achievement $\dagger$ & -0.058 & 0.297 & $(-0.223,0.107)$ & 0.488 \\
\hline \multicolumn{5}{|c|}{ Academic performance (school grades) } \\
\hline Mathematics $\ddagger$ & -0.176 & 0.183 & $(-0.383,0.026)$ & 0.086 \\
\hline Spanish language $\neq$ & -0.188 & 0.190 & $(-0.395,0.012)$ & 0.065 \\
\hline English $\ddagger$ & -0.168 & 0.149 & $(-0.379,0.038)$ & 0.107 \\
\hline Natural and social science $\dagger$ & -0.226 & 0.250 & $(-0.427,-0.031)$ & 0.024 \\
\hline Grade point average $\ddagger$ & -0.269 & 0.238 & $(-0.470,-0.076)$ & 0.007 \\
\hline \multicolumn{5}{|l|}{ Brain volumes } \\
\hline Total gray matter $\dagger \dagger$ & 0.058 & 0.329 & $(-0.112,0.225)$ & 0.505 \\
\hline Total white matter $\neq \neq$ & -0.058 & 0.450 & $(-0.212,0.097)$ & 0.462 \\
\hline Total brain volume $\dagger \dagger$ & 0.018 & 0.373 & $(-0.145,0.181)$ & 0.828 \\
\hline \multicolumn{5}{|l|}{ Hippocampal GMV } \\
\hline Right hippocampus $\dagger \dagger \dagger$ & -0.090 & 0.127 & $(-0.277,0.098)$ & 0.345 \\
\hline Left hippocampus $\neq+\ddagger$ & 0.004 & 0.046 & $(-0.191,0.199)$ & 0.969 \\
\hline 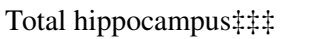 & -0.043 & 0.079 & $(-0.235,0.148)$ & 0.655 \\
\hline
\end{tabular}

The predictor variable was introduced in separate hierarchical regression models. Potential confounders (i.e., age, sex, maternal education level, paternal education level, wave, and peak height velocity) were included into step 1 of the stepwise regression to test their association to the outcomes so that for each outcome, only the relevant confounders were retained in the final models using the method STEPWISE. In step 2, the exposure variable of interest, SRBD scale was entered using the method ENTER to force to be in the model. $\beta$ values are standardized coefficients. Bolded font indicates that the specific association surpassed the Benjamini-Hochberg correction for multiple comparisons test (performed for each domain, i.e., academic performance assessed by Woodcock-Muñoz battery, academic performance assessed by school grades, brain volumes, and hippocampal GMV). $\dagger$ Adjusted by age and maternal education level. $\ddagger$ Adjusted by maternal education level. $\dagger \dagger$ Adjusted by sex and paternal education level. $\$$ \$Adjusted by age, sex, paternal education level, and peak height velocity. $\dagger \dagger \dagger$ Adjusted by sex and peak height velocity. $\$+\$$ Adjusted by sex. $S D B$ sleep-disordered breathing, $S R B D$ sleep-related breathing disorders, $G M V$ gray matter volume primarily explained by the different constructs that were measured, since Biggs et al. [19] focused on school functioning as a measure related to quality of life, yet they did not collect school grades. Moreover, recent studies found that overweight/obesity was inversely associated with school performance in childhood [54, 55]. Indeed, both SDB and obesity may adversely influence cognitive functioning [20] and, subsequently, academic performance. Children with overweight/obesity and SDB were at higher risk of neurocognitive deficits than normal-weight children (with and without SDB) [14, 15]. As an alternative explanation, they suggested that obesity might worsen neurocognitive performance in children rather than SDB alone, yet they did not include a non-SDB overweight/obesity group. Hence, we added to the literature by showing that SDB symptoms may worsen already-impaired academic achievement in children with overweight/obesity. However, evidence that affirms the association of obesity with academic performance is not strong enough [56], and future research with a healthy-weight control group and proper sample size should further investigate the interactions between obesity and SDB in association with academic performance.

Taken together, our findings and the abovementioned studies suggested that the previously observed association between SDB and school grades in normal-weight children $[7-10,57,58]$ is also apparent in children with overweight or obesity. The mechanisms driving the association between SDB and school performance remain unclear [18]. SDB may produce intermittent hypoxia, hypercapnia, and reoxygenation, along with changes in cerebral blood flow and sleep fragmentation [59], potentially affecting cognitive functioning. Although variability in cognitive function in children with SDB may be explained by a reduction in cerebral oxygenation [60], the pathophysiology of the involvement of the central nervous system during OSA is still debated [61]. However, a recent review found that cerebral oxygenation levels were maintained in this population [34] and were not related to cognitive deficits, but they were associated with 
Table 3 Hierarchical linear regression for the associations of SDB subscales (snoring, sleepiness, and inattention/ hyperactivity) with academic performance, brain volumes, and hippocampal gray matter volume variables

\begin{tabular}{|c|c|c|c|c|c|c|}
\hline & \multicolumn{2}{|c|}{ Snoring } & \multicolumn{2}{|c|}{ Daytime sleepiness } & \multicolumn{2}{|c|}{$\begin{array}{l}\text { Inattention/ } \\
\text { hyperactivity }\end{array}$} \\
\hline & $\boldsymbol{\beta}$ & $\boldsymbol{P}$ & $\boldsymbol{\beta}$ & $\boldsymbol{P}$ & $\boldsymbol{\beta}$ & $P$ \\
\hline \multicolumn{7}{|c|}{ Academic performance (Woodcock-Muñoz) } \\
\hline Mathematics $\dagger$ & 0.060 & 0.493 & -0.086 & 0.326 & 0.028 & 0.747 \\
\hline Reading $\neq$ & 0.027 & 0.751 & -0.124 & 0.146 & -0.025 & 0.768 \\
\hline Writing $\neq$ & -0.032 & 0.720 & -0.168 & 0.059 & -0.165 & 0.070 \\
\hline Total achievement $\dagger$ & 0.029 & 0.726 & -0.154 & 00.063 & -0.056 & 0.506 \\
\hline \multicolumn{7}{|c|}{ Academic performance (school grades) } \\
\hline Mathematics $\ddagger$ & 0.075 & 0.461 & -0.138 & 0.181 & -0.257 & 0.014 \\
\hline Spanish language & 0.015 & 0.886 & -0.094 & 0.365 & -0.182 & 0.083 \\
\hline English $¥$ & 0.036 & 0.731 & -0.105 & 0.320 & -0.145 & 0.180 \\
\hline Natural and social science $\dagger$ & 0.029 & 0.771 & -0.204 & 0.043 & -0.253 & 0.013 \\
\hline Grade point average $\ddagger$ & -0.004 & 0.970 & -0.141 & 0.171 & -0.297 & 0.004 \\
\hline \multicolumn{7}{|l|}{ Brain volumes } \\
\hline Total gray matter $\dagger \dagger$ & -0.007 & 0.935 & -0.006 & 0.945 & 0.132 & 0.130 \\
\hline Total white matterł末 & 0.035 & 0.657 & -0.087 & 0.267 & 0.020 & 0.800 \\
\hline Total brain volume $\dagger \dagger$ & 0.026 & 0.749 & -0.046 & 0.579 & 0.102 & 0.225 \\
\hline \multicolumn{7}{|l|}{ Hippocampal GMV } \\
\hline Right hippocampus $\dagger+\dagger$ & -0.133 & 0.164 & -0.020 & 0.833 & -0.090 & 0.349 \\
\hline Left hippocampus $+\neq \ddagger$ & -0.071 & 0.471 & -0.113 & 0.255 & 0.026 & 0.791 \\
\hline Total hippocampus抹 & -0.096 & 0.320 & -0.080 & 0.415 & -0.033 & 0.735 \\
\hline
\end{tabular}

The predictor variable was introduced in separate hierarchical regression models. Potential confounders (i.e., age, sex, maternal education level, paternal education level, wave, and peak height velocity) were included into step 1 of the stepwise regression to test their association to the outcomes so that for each outcome, only the relevant confounders were retained in the final models using the method STEPWISE. In step 2, the exposure variable of interest (e.g., snoring or sleepiness, or inattention/hyperactivity subscale) was entered using the method ENTER to force to be in the model. $\beta$ values are standardized coefficients. Bolded font indicates that the specific association surpassed the Benjamini-Hochberg correction for multiple comparisons test (performed for each domain, i.e., academic performance assessed by WoodcockMuñoz battery, academic performance assessed by school grades, brain volumes, and hippocampal GMV). $\dagger$ Adjusted by age and maternal education level. $\$$ Adjusted by maternal education level. $† \dagger$ Adjusted by sex and paternal education level. $\$$ Adjusted by age, sex, paternal education level and peak height velocity. $\dagger \dagger \dagger$ Adjusted by sex and peak height velocity. $\$+\$$ Adjusted by sex. SDB sleep-disordered breathing, GMV gray matter volume behavioral problems in children with SDB [62]. This conclusion may explain the association in our study between SDB and school grades, where children's behavior, general functioning, and teachers' influence on children's behaviors are relevant. Future studies exploring SDB severity with polysomnography and a proper sample size should corroborate or contrast with our findings.

With regard to brain structure, SDB severity was not related to total brain volume or specifically to hippocampal volume in our sample of children with overweight/obesity. Similarly, a study in 10-year-old children found no significant difference between the OSA and control children in total and regional brain volume and density (i.e., GMV, gray/white matter ratio, total brain volume, and gray/total brain ratio) [35]. Unlike our study, a recent review identified that SDB was associated with changes in the brain using MRI in children with normal weight [34]. Likewise, using polysomnography, recent studies determined that normal-weight children (7-11 years) with OSA had smaller regional GMV [32] and WMV [63] than children without OSA. Furthermore, Walter and Horne [33] concluded in a review that previous MRI studies found significant structural changes (i.e., reduced white and gray matter of particular brain areas, including the hippocampus) in children with SDB. Our results may differ from these findings due to the method of measuring SDB (i.e., overnight polysomnography vs. PSQ). It is important to consider that SDB severity in our participants may be mild (i.e., the SRBD average score was $0.19[\mathrm{SD}=0.13]$ on a scale with a range from 0 to 1 , with 0.33 indicating higher risk for SDB). Other studies in children with higher SDB severity have found regional gray matter deficits [35]. The lower SDB severity in our participants could explain the lack of apparent brain volume deficits and the small-sized associations with academic achievement. 
The lack of significant associations may be due to the low statistical power reached in this analysis. Only 17 participants presented SDB in this study (15.6\%). As such, SDB severity might not have been sufficient to affect myelination and neuronal connectivity in our participants [64]. However, it is difficult to compare our findings to other cohorts since no previous study was conducted with children with overweight/obesity. In adults with OSA, lower GMV in the left hippocampus [65] and in the right hippocampus $[66,67]$ has been found, although this association was not observed here in the context of childhood obesity. Differing developmental stages [68] and not using polysomnography in our study may have partly explained the lack of observed associations. Thus, larger studies including both normal-weight children and children with overweight/obesity are needed to confirm these findings.

The high prevalence of pediatric obesity worldwide and its associated health-related problems, such as SDB, provide an impetus for the investigation of brain health factors associated with this disorder. Previous research has shown links between SDB and academic performance and brain structure in the pediatric population. However, information on how SDB severity in children with overweight/obesity is associated with academic performance and brain health is limited. Our study contributes to the current knowledge by suggesting that SDB may worsen academic performance in children with overweight/obesity (who often show impaired performance). We also provided a broader picture of academic performance by including two different assessments: school grades and standardized test results. Although we did not find associations with brain structures, it remains to be elucidated whether these changes in school grades have any clinical significance. Some limitations need to be acknowledged. First, this was a cross-sectional study; hence, it does not allow for causal inference for any of the observed associations. Second, our data were obtained from a parent-reported questionnaire instead of polysomnographic data (i.e., the gold standard for the detection of SDB and its severity); however, this questionnaire is valid and reliable for the diagnosis of SDB symptoms. Third, including a group of children with normal weight could be informative toward understanding the interaction between obesity and SDB. Unfortunately, we did not collect data in children with normal weight, and we encourage future researchers to gain insight into this interaction by assessing children with varied weight status. Fourth, teachers may have graded the children from different schools differently. Fifth, our sample size and the prevalence of SDB may have limited the statistical power of the analysis. Future research should confirm our findings. Caution is advised with the extrapolation of these findings to younger children or to other determinants of SDB that were not considered in this study (e.g., adenotonsillar disease, ethnicity, and levels of deprivation). On the other hand, the strengths of this study include the use of MRI for the quantification of GMV in the hippocampus and total brain volume; the focus on children with overweight/obesity, who are particularly vulnerable to sleep disorders; and a comprehensive academic performance assessment using two different methods, i.e., standardized tests and school grades.

\section{Conclusion}

Our study contributes to the literature by indicating that SDB severity was related to lower academic performance at school in children with overweight/obesity, which was driven by inattention/hyperactivity. However, we did not observe associations with academic performance measured by a standardized test or brain structure in children with overweight/obesity. Accordingly, our findings suggest that SDB symptoms may adversely affect learning in school in children with overweight/obesity beyond the effects of weight status alone.

Authors' contributions Ms Torres-Lopez drafted the initial manuscript, conducted all statistical analyses, and reviewed and revised the manuscript. Drs. Cadenas-Sanchez and Migueles collected data, carried out the initial analyses, and reviewed and revised the manuscript. Drs. Esteban-Cornejo, Molina-Garcia, Hillman, and Catena critically reviewed the manuscript for important intellectual content. Dr. Ortega conceptualized and designed the study, coordinated and supervised data collection, and critically reviewed and revised the manuscript. All authors read and approved the final manuscript as submitted and agree to be accountable for all aspects of the work.

Funding Funding for open access charge: Universidad de Granada / CBUA. This work is part of a PhD thesis conducted in the Official Doctoral Programme in Biomedicine of the University of Granada, Spain. Preliminary data from this manuscript has been presented previously in The International Society of Behavioral Nutrition and Physical Activity (ISBNPA) Xchange 2021 Annual Meeting. The ActiveBrains project was funded by the Spanish Ministry of Economy and Competitiveness and the "Fondo Europeo de Desarrollo Regional (FEDER)" (DEP201347540, DEP2016-79512-R, DEP2017-91544-EXP and RYC-201109011). L.V.T.-L. is supported by a Grant from the Spanish Ministry of Science, Innovation and Universities (FPU17/04802). J.H.M. is supported by a Grant from the Spanish Ministry of Education, Culture and Sport (FPU15/02645). C.C.-S. is supported by the Spanish Ministry of Science and Innovation (FJC2018-037925-I). IEC is supported by the Spanish Ministry of Science and Innovation (RYC2019-027287-I). Additional support was obtained from the University of Granada, Plan Propio de Investigación 2016, Excellence actions: Units of Excellence, Scientific Excellence Unit on Exercise and Health (UCEES), by the Junta de Andalucía, Consejería de Conocimiento, Investigación y Universidades, and European Regional Development Funds (ref. SOMM17/6107/UGR). In addition, funding was provided by the SAMID III network, RETICS, funded by the PNI + D + I 2017-2021 (Spain), ISCIII- Sub-Directorate General for Research Assessment and Promotion, the European Regional Development Fund (ERDF) (Ref. RD16/0022), the EXERNET Research Network on Exercise and Health (DEP2005-00046/ACTI; 09/UPB/19; 45/UPB/20; 27/UPB/21), the European Union's 2020 research and innovation program under grant 
agreement No.667302, and the HL-PIVOT network-Healthy Living for Pandemic Event Protection. Additional funding was obtained from the Andalusian Operational Programme supported with European Regional Development Funds (ERDF in English, FEDER in Spanish, project ref: B-CTS-355-UGR18).

Availability of data and material Not applicable.

Code availability Not applicable.

\section{Declarations}

Ethics approval This study was conducted according to the Declaration of Helsinki. The study protocol was approved by the Ethics Committee on Human Research (CEIH) of the University of Granada and registered in ClinicalTrials.gov (identifier: NCT02295072).

Consent to participate Legal guardians were informed of the purpose of the study and written informed consent for all participants was obtained.

Consent for publication All parents or legal guardians have signed informed consent regarding publishing data about their children.

Conflict of interest The authors declare no competing interests.

Open Access This article is licensed under a Creative Commons Attribution 4.0 International License, which permits use, sharing, adaptation, distribution and reproduction in any medium or format, as long as you give appropriate credit to the original author(s) and the source, provide a link to the Creative Commons licence, and indicate if changes were made. The images or other third party material in this article are included in the article's Creative Commons licence, unless indicated otherwise in a credit line to the material. If material is not included in the article's Creative Commons licence and your intended use is not permitted by statutory regulation or exceeds the permitted use, you will need to obtain permission directly from the copyright holder. To view a copy of this licence, visit http://creativecommons.org/licenses/by/4.0/.

\section{References}

1. Gokdemir Y, Ersu R (2016) Sleep disordered breathing in childhood. Eur Respir Rev 25:48-53. https://doi.org/10.1183/16000617. 0081-2015

2. Kaditis AG, Alvarez MLA, Boudewyns A et al (2016) Obstructive sleep disordered breathing in 2- to 18-year-old children: diagnosis and management. Eur Respir J 47:69-94. https://doi.org/10.1183/ 13993003.00385-2015

3. Lumeng JC, Chervin RD (2008) Epidemiology of pediatric obstructive sleep apnea. Proc Am Thorac Soc 5:242-252

4. Abazi Y, Cenko F, Cardella M et al (2020) Sleep disordered breathing: an epidemiological study among albanian children and adolescents. Int J Environ Res Public Health 17:1-11. https://doi. org/10.3390/ijerph17228586

5. Verhulst SL, Van Gaal L, De Backer W, Desager K (2008) The prevalence, anatomical correlates and treatment of sleep-disordered breathing in obese children and adolescents. Sleep Med Rev 12:339-346

6. Mitchell RB, Garetz S, Moore RH et al (2015) The use of clinical parameters to predict obstructive sleep apnea syndrome severity in children: the Childhood Adenotonsillectomy (CHAT) Study Randomized Clinical Trial HHS Public Access. JAMA Otolaryngol Head Neck Surg 141:130-136. https://doi.org/10.1001/jamaoto.2014.3049
7. Galland B, Spruyt K, Dawes P et al (2015) Sleep disordered breathing and academic performance: a meta-analysis. Pediatrics 136:e934-e946. https://doi.org/10.1542/peds.2015-1677

8. Gatica D, Rodríguez-Núñez I, Zenteno D et al (2017) Association between sleep-related breathing disorders and academic performance among children from Concepción. Chile Br reports / Arch Argent Pediatr 115. https://doi.org/10.5546/aap.2017.eng.497

9. Harding R, Haszard JJ, Schaughency E et al (2020) Parent report of children's sleep disordered breathing symptoms and limited academic progress in reading, writing, and math. Sleep Med 65:105-112. https://doi.org/10.1016/j.sleep.2019.07.018

10. Liu J, Liu X, Ji X et al (2016) Sleep disordered breathing symptoms and daytime sleepiness are associated with emotional problems and poor school performance in children. Psychiatry Res 242:218-225. https://doi.org/10.1016/j.psychres.2016.05.017

11. Kohler M (2010) Neurocognitive performance and behavior before and after treatment for sleep-disordered breathing in children. Nat Sci Sleep 2:159. https://doi.org/10.2147/NSS.S6934

12. Marcus CL, Moore RH, Rosen CL et al (2013) A randomized trial of adenotonsillectomy for childhood sleep apnea. N Engl J Med 368:2366-2376. https://doi.org/10.1056/NEJMOA1215881/ SUPPL_FILE/NEJMOA1215881_DISCLOSURES.PDF

13. Harding R, Schaughency E, Haszard JJ et al (2021) Sleep-related breathing problem trajectories across early childhood and academic achievement-related performance at age eight. Front Psychol 12. https://doi.org/10.3389/fpsyg.2021.661156

14. Kohler M, Kennedy D, Martin J et al (2018) The influence of body mass on long-term cognitive performance of children treated for sleep-disordered breathing. Sleep Med 51:1-6. https://doi.org/10. 1016/j.sleep.2018.05.021

15. Vitelli O, Tabarrini A, Miano S et al (2015) Impact of obesity on cognitive outcome in children with sleep-disordered breathing. Sleep Med 16:625-630. https://doi.org/10.1016/j.sleep.2014.12. 015

16. Rhodes SK, Shimoda KC, Waid LR et al (1995) Neurocognitive deficits in morbidly obese children with obstructive sleep apnea. J Pediatr 127:741-744. https://doi.org/10.1016/S0022-3476(95) 70164-8

17. Tan E, Healey D, Schaughency E et al (2014) Neurobehavioural correlates in older children and adolescents with obesity and obstructive sleep apnoea. J Paediatr Child Health 50:16-23. https://doi.org/10.1111/jpc.12390

18. Beebe DW, Douglas Ris; M, Kramer ME et al (2010) The association between sleep disordered breathing, academic grades, and cognitive and behavioral functioning among overweight subjects during middle to late childhood. Sleep 33:1447-1456

19. Biggs SN, Tamanyan K, Walter LM et al (2017) Overweight and obesity add to behavioral problems in children with sleep-disordered breathing. Sleep Med 39:62-69. https://doi.org/10.1016/j.sleep. 2017.09.001

20. Spruyt K, Gozal D (2012) A mediation model linking body weight, cognition, and sleep-disordered breathing. Am J Respir Crit Care Med 185:199-205. https://doi.org/10.1164/rccm. 201104-0721OC

21. Erickson KI, Hillman C, Stillman CM et al (2019) Physical activity, cognition, and brain outcomes: a review of the 2018 physical activity guidelines. Med Sci Sports Exerc 51:1242-1251. https:// doi.org/10.1249/MSS.0000000000001936

22. Donnelly JE, Hillman CH, Castelli D et al (2016) Physical activity, fitness, cognitive function, and academic achievement in children: a systematic review. Med Sci Sports Exerc 48:1197-1222. https:// doi.org/10.1249/MSS.0000000000000901

23. Dudai Y, Karni A, Born J (2015) The consolidation and transformation of memory. Neuron 88:20-32. https://doi.org/10.1016/j. neuron.2015.09.004 
24. Axmacher N, Draguhn A, Elger CE, Fell J (2009) Memory processes during sleep: Beyond the standard consolidation theory. Cell Mol Life Sci 66:2285-2297. https://doi.org/10.1007/ s00018-009-0019-1

25. Halbower AC, Degaonkar M, Barker PB et al (2006) Childhood obstructive sleep apnea associates with neuropsychological deficits and neuronal brain injury. PLoS Med 3:1391-1402. https:// doi.org/10.1371/journal.pmed.0030301

26. Kheirandish-Gozal L, Yoder K, Kulkarni R et al (2014) Preliminary functional mri neural correlates of executive functioning and empathy in children with obstructive sleep apnea. Sleep 37:587592. https://doi.org/10.5665/sleep.3504

27. Lv J, Shi L, Zhao L et al (2017) Morphometry analysis of basal ganglia structures in children with obstructive sleep apnea. J Xray Sci Technol 25:93-99. https://doi.org/10.3233/XST-16171

28. Cha J, Zea-Hernandez JA, Sin S et al (2017) The effects of obstructive sleep apnea syndrome on the dentate gyrus and learning and memory in children. J Neurosci 37:4280-4288. https://doi. org/10.1523/JNEUROSCI.3583-16.2017

29. Horne RSC, Roy B, Walter LM et al (2018) Regional brain tissue changes and associations with disease severity in children with sleep-disordered breathing. Sleep 41. https://doi.org/10.1093/ sleep/zsx203

30. Macey PM, Kheirandish-Gozal L, Prasad JP et al (2018) Altered regional brain cortical thickness in pediatric obstructive sleep apnea. Front Neurol 9:22. https://doi.org/10.3389/fneur.2018. 00004

31. Kheirandish-Gozal L, Sahib AK, Macey PM et al (2018) Regional brain tissue integrity in pediatric obstructive sleep apnea. Neurosci Lett 682:118-123. https://doi.org/10.1016/j.neulet.2018.06. 002

32. Philby MF, Macey PM, Ma RA et al (2017) Reduced regional grey matter volumes in pediatric obstructive sleep apnea. Sci Rep 7. https://doi.org/10.1038/srep44566

33. Walter LM, Horne RSC (2018) Obstructive sleep-disordered breathing in children: Impact on the developing brain. Pediatr Respirol Crit Care Med 2:58-64. https://doi.org/10.4103/prcm. prom_16_18

34. Walter LM, Shepherd KL, Yee A, Horne RSC (2020) Insights into the effects of sleep disordered breathing on the brain in infants and children: imaging and cerebral oxygenation measurements. Sleep Med Rev 50

35. Chan KC, Shi L, So HK et al (2014) Neurocognitive dysfunction and grey matter density deficit in children with obstructive sleep apnoea. Sleep Med 15:1055-1061. https://doi.org/10.1016/j.sleep. 2014.04.011

36. Celle S, Delon-Martin C, Roche F et al (2016) Desperately seeking grey matter volume changes in sleep apnea: a methodological review of magnetic resonance brain voxel-based morphometry studies. Sleep Med Rev 25:112-120. https://doi.org/10.1016/j. smrv.2015.03.001

37. Su M-S, Zhang H-L, Cai X-H et al (2016) Obesity in children with different risk factors for obstructive sleep apnea: a communitybased study. Eur J Pediatr 175:211-220. https://doi.org/10.1007/ s00431-015-2613-6

38. Ou X, Andres A, Pivik RT et al (2015) Brain gray and white matter differences in healthy normal weight and obese children. J Magn Reson Imaging 42:1205-1213. https://doi.org/10.1002/ jmri.24912

39. Cadenas-Sánchez C, Mora-González J, Migueles JH et al (2016) An exercise-based randomized controlled trial on brain, cognition, physical health and mental health in overweight/obese children (ActiveBrains project): rationale, design and methods. Contemp Clin Trials 47:315-324. https://doi.org/10.1016/j.cct.2016.02.007

40. Cole TJ, Lobstein T (2012) Extended international (IOTF) body mass index cut-offs for thinness, overweight and obesity. Pediatr
Obes 7:284-294. https://doi.org/10.1111/j.2047-6310.2012. 00064.x

41. Bervoets L, Massa G (2014) Defining morbid obesity in children based on BMI 40 at age 18 using the extended international (IOTF) cut-offs. Pediatr Obes 9:e94-e98. https://doi.org/10. 1111/j.2047-6310.2014.00217.x

42. Tanner JM, Whitehouse RH (1976) Clinical longitudinal standards for height, weight, height velocity, weight velocity, and stages of puberty. Arch Dis Child 51:170-179. https://doi.org/10.1136/ ADC.51.3.170

43. Vila MT, Torres AM, Soto BB (2007) Spanish version of the Pediatric Sleep Questionnaire (PSQ). A useful instrument in investigation of sleep disturbances in childhood. Reliability analysis An Pediatr 66:121-128

44. Chervin RD, Weatherly RA, Garetz SL et al (2007) Pediatric sleep questionnaire. Prediction of sleep apnea and outcomes. Arch Otolaryngol Head Neck Surg 133:216-222. https://doi.org/10.1001/ archotol.133.3.216

45. Chervin RD, Hedger K, Dillon JE, Pituch KJ (2000) Pediatric sleep questionnaire (PSQ): Validity and reliability of scales for sleep-disordered breathing, snoring, sleepiness, and behavioral problems. Sleep Med 1:21-32. https://doi.org/10.1016/S13899457(99)00009-X

46. Torres-Lopez LV, Cadenas-Sanchez C, Migueles JH et al (2020) Associations of sedentary behaviour, physical activity, cardiorespiratory fitness and body composition with risk of sleep-related breathing disorders in children with overweight/obesity: a cross-sectional study. J Clin Med 9:1544. https://doi.org/10.3390/jcm9051544

47. Michelet D, Julien-Marsollier F, Vacher T et al (2019) Accuracy of the sleep-related breathing disorder scale to diagnose obstructive sleep apnea in children: a meta-analysis. Sleep Med 54:78-85

48. McGrew K, Woodcock R (2001) Woodcock-Johnson III: Technical Manual. Riverside Publishing Company, Itasca, IL

49. Esteban-Cornejo I, Cadenas-Sanchez C, Contreras-Rodriguez O et al (2017) A whole brain volumetric approach in overweight/obese children: examining the association with different physical fitness components and academic performance. The ActiveBrains project Neuroimage 159:346-354. https://doi.org/10.1016/j.neuroimage. 2017.08.011

50. Moore SA, McKay HA, Macdonald H et al (2015) Enhancing a somatic maturity prediction model. Med Sci Sports Exerc 47:1755-1764. https://doi.org/10.1249/MSS.0000000000000588

51. Blom G (1958) Statistical estimates and transformed beta variables. Wiley, New York, NY

52. Benjamini Y, Hochberg Y (1995) Controlling the false discovery rate: a practical and powerful approach to multiple testing. J R Stat Soc Ser B 57:289-300. https://doi.org/10.2307/2346101

53. Willingham W, Pollack J, Lewis C (2002) Grades and test scores: accounting for observed differences. J Educ Meas 39:1-37

54. Esteban-Cornejo I, Tejero-González CM, Castro-Piñero J et al (2015) Independent and combined influence of neonatal and current body composition on academic performance in youth: the UP \& DOWN study. Pediatr Obes 10:157-164. https://doi.org/10. 1111/IJPO.239

55. Sardinha LB, Marques A, Martins S et al (2014) Fitness, fatness, and academic performance in seventh-grade elementary school students. BMC Pediatr 14:1-9. https://doi.org/10.1186/14712431-14-176/FIGURES/1

56. Santana CCA, Hill JO, Azevedo LB et al (2017) The association between obesity and academic performance in youth: a systematic review. Obes Rev 18:1191-1199. https://doi.org/10.1111/obr. 12582

57. Goyal A, Pakhare AP, Bhatt GC et al (2018) Association of pediatric obstructive sleep apnea with poor academic performance: a school-based study from India. Lung India 35:132-136. https:// doi.org/10.4103/lungindia.lungindia_218_17 
58. Brockmann PE, Bertrand P, Pardo T et al (2012) Prevalence of habitual snoring and associated neurocognitive consequences among Chilean school aged children. Int J Pediatr Otorhinolaryngol 76:1327-1331. https://doi.org/10.1016/j.ijporl.2012.05.028

59. Rosenzweig I, Glasser M, Polsek D et al (2015) Sleep apnoea and the brain: a complex relationship. Lancet Respir Med 3:404-414

60. Khadra MA, McConnell K, VanDyke R et al (2008) Determinants of regional cerebral oxygenation in children with sleep-disordered breathing. Am J Respir Crit Care Med 178:870-875. https://doi. org/10.1164/rccm.200802-3210C

61. Luo YG, Wang D, Liu K et al (2015) Brain structure network analysis in patients with obstructive sleep apnea PLoS One 10. https:// doi.org/10.1371/journal.pone.0139055

62. Tamanyan K, Walter LM, Weichard A et al (2018) Age effects on cerebral oxygenation and behavior in children with sleep-disordered breathing. Am J Respir Crit Care Med 197:1468-1477. https://doi. org/10.1164/rccm.201709-1825OC

63. Musso MF, Lindsey HM, Wilde EA et al (2020) Volumetric brain magnetic resonance imaging analysis in children with obstructive sleep apnea. Int J Pediatr Otorhinolaryngol 138:110369. https://doi. org/10.1016/j.ijporl.2020.110369

64. Brockmann PE, Alonso-Álvarez ML, Gozal D (2018) Diagnosing sleep apnea-hypopnea syndrome in children: past, present, and future. Arch Bronconeumol English Ed 54:303-305. https://doi. org/10.1016/j.arbr.2018.04.004

65. Morrell MJ, McRobbie DW, Quest RA et al (2003) Changes in brain morphology associated with obstructive sleep apnea. Sleep Med 4:451-454. https://doi.org/10.1016/S1389-9457(03)00159-X

66. Tahmasian M, Rosenzweig I, Eickhoff SB et al (2016) Structural and functional neural adaptations in obstructive sleep apnea: an activation likelihood estimation meta-analysis. Neurosci Biobehav Rev 65:142-156. https://doi.org/10.1016/j.neubiorev.2016.03.026

67. Torelli F, Moscufo N, Garreffa G et al (2011) Cognitive profile and brain morphological changes in obstructive sleep apnea. Neuroimage 54:787-793. https://doi.org/10.1016/j.neuroimage.2010. 09.065

68. Lenroot RK, Gogtay N, Greenstein DK et al (2007) Sexual dimorphism of brain developmental trajectories during childhood and adolescence. Neuroimage 36:1065-1073. https://doi.org/10.1016/j. neuroimage.2007.03.053

Publisher's Note Springer Nature remains neutral with regard to jurisdictional claims in published maps and institutional affiliations.

\section{Authors and Affiliations}

\section{Lucia V. Torres-Lopez ${ }^{1}$ (1) . Cristina Cadenas-Sanchez ${ }^{1,2}$. Jairo H. Migueles ${ }^{3,1,4} \cdot$ Irene Esteban-Cornejo ${ }^{1}$. Pablo Molina-Garcia ${ }^{1}$. Charles H. Hillman ${ }^{5}$. Andres Catena ${ }^{6}$. Francisco B. Ortega ${ }^{1,7,4}$}

Cristina Cadenas-Sanchez

cristina.cadenas.sanchez@gmail.com

Jairo H. Migueles

jairo.hidalgo.migueles@liu.se

Irene Esteban-Cornejo

ireneesteban@ugr.es

Pablo Molina-Garcia

pablomolinag5@gmail.com

Charles H. Hillman

c.hillman@northeastern.edu

Andres Catena

acatenam@gmail.com

1 PROFITH "PROmoting FITness and Health Through Physical Activity" Research Group, Department of Physical Education and Sports, Faculty of Sport Sciences, Sport and Health University Research Institute (iMUDS), University of Granada, Granada 18011, Spain
2 Institute for Innovation and Sustainable Development in the Food Chain (IS-FOOD), Public University of Navarra, Pamplona, Spain

3 Department of Health, Medicine and Caring Sciences, Linköping University, Linköping, Sweden

4 Department of Biosciences and Nutrition, Karolinska Institutet, Huddinge 14183, Sweden

5 Center for Cognitive and Brain Health, Department of Psychology, Department of Physical Therapy, Movement, and Rehabilitation Sciences, Northeastern University, Boston, MA, USA

6 School of Psychology, University of Granada, Granada, Spain

7 Faculty of Sport and Health Sciences, University of Jyväskylä, Jyväskylä, Finland 